\title{
Jüdisch-europäische Erinnerungskultur im Zeitalter des Kulturwandels
}

\author{
Habib Tekin (D), İstanbul \\ https://doi.org/10.37583/diyalog.1030310
}

\begin{abstract}
Deutsch)
Im 21. Jahrhundert stehen Medien, insbesondere soziale Medien und deren Einfluss auf Kinder und Jugendliche zunehmend im Zentrum des gesellschaftlichen Lebens. Sozialen Medien wird eo ipso eine wichtige Funktion in der individuellen Meinungsbildung wie auch gesellschaftlichen Rollenvergabe zugesprochen. Im Rahmen des vorliegenden Vortrags sollen auf Grundlage des theoretischen Ansatzes der Medialisierung folglich auch die gesellschaftlichen Veränderungen und der soziale Wandel vor dem Hintergrund der medialen Omnipräsenz dargestellt werden. Dabei wird der Versuch unternommen, einen (sozial)medialen Korpus der jüdisch-europäischen Erinnerungskultur zu erstellen. Das angedachte Korpus setzt sich aus der ARD und ZDF-Mediathek zusammen. Exemplarisch wird die Zukunftspodcast der Tagesschau mal angenommen. Keine Zeitzeugen mehr? Was dann? vorgestellt. Die vorgestellten Korpora sind ferner als eine Form der bezeugten Berichterstattung zu verstehen, die ein Gedenken an den Holocaust und die Gräueltaten auch in Zukunft wachhalten sollen. Neben den (deutsch-)jüdischen Studien, der deutsch-jüdischen Literatur und der wissenschaftlichen Auseinandersetzung mit der Thematik, lassen sich medialen Aufzeichnungen in Form von Video- oder Audiomaterial eine große Bedeutung zuweisen. In der Hoffnung, dass sich Geschichte nicht wiederholt, übernehmen mediale Korpora in diesem Sinne eine wichtige Funktion in der Aufrechterhaltung der Erinnerung an die Verbrechen des NS-Regimes und dem Verbleib im kollektiven Gedächtnis. Demnach können mediale Korpora in Form bezeugter Berichterstattung ein aktives Engagement im Akt des Erinnerns anregen und die Sensibilität im Hinblick auf etwaige zukünftige Verbrechen fördern.

Das Ziel des Beitrags besteht demzufolge darin, die Entstehung, Erstellung und Funktion eines möglichen (sozial)medialen Korpus' der jüdisch-europäischen Erinnerungskultur zu skizzieren. Darüber hinaus werden informative Diskursmöglichkeiten und weitere Anregungen für die Implementierung einer digitalisierten Erinnerungskultur in Schulen und anderen Bildungsinstitutionen gegeben.
\end{abstract}

Schlüsselwörter: Holocaust, Kulturwandel, Anne Frank Lernlabor, Hologramm, digitale Zeugnisse.

\section{Abstract (English)}

\section{Jewish-European Culture of Remembrance in The Age of Cultural Change}

In the 21 st century, the media, especially social media and their influence on children and young people, are increasingly at the center of social life. Social media is (eo ipso) assigned an important function in the formation of individual opinions as well as the allocation of social roles. In the context of the present paper, on the basis of the theoretical approach of medialization, social change should be presented against the background of media omnipresence. The attempt is made to create a (social) media corpus of the JewishEuropean culture of remembrance. The envisioned corpus consists of the ARD and ZDF media library. The 
future podcast of the Tagesschau mal angenommen. Keine Zeitzeugen mehr? Was dann? is presented. The given corpora are also to be understood as a form of witnessed reporting that is intended to keep the memory of the Holocaust and the atrocities alive in the future. In addition to (German-) Jewish studies, GermanJewish literature and the scientific researches of that topic, media recordings in the form of video or audio material can be assigned great importance. In the hope that history does not repeat itself, media corpora take on an important function in maintaining the memory of the atrocities of the Nazi regime and keeping alive the collective memory of the Jewish-European culture. Thus, media corpora in the form of witnessed reporting can stimulate active engagement in the act of remembering and raise social awareness of possible future crimes.

The aim of the article is therefore to outline the emergence, creation and function of a possible (social) media corpus of the Jewish-European culture of remembrance. In addition, informative discourse opportunities and further suggestions for the implementation of a digitized culture of remembrance in schools and other educational institutions are given.

Keywords: Holocaust, Cultural Change, Anne Frank Lernlabor, Hologram, digital testimonies. 


\section{EXTENDED ABSTRACT}

Annelies Marie Frank (June 12, 1929-1947 in the Bergen-Belsen concentration camp) is a young girl from Frankfurt am Main, who is ascribed an important function in German-Jewish literature and in the European culture of remembrance, especially as an individual in childhood. Her writing is so important because she looks into the horror from the perspective of a child and reproduces her experiences, fears, wishes and ideas for the future, again and again in view of the naivety and purity of a child. Her diary is part of the literary canon of German-Jewish literature of the 20th century, which enables a confrontation and a view into the horror of the National Socialist dictatorship. One would dare to say that being a human is her only wish. This type of written form of remembrance is important in the culture of remembrance.

Beyond that, there are those who survived the horror and are therefore also referred to as Holocaust survivors. These in turn make their contribution to sensitizing people on all social, societal and political levels so that - to use the words of Adorno - the "barbarism" does not repeat itself. Above all, public channels such as ARD and ZDF primarily, but also newspapers and magazines contribute to raising awareness within the society and allow Holocaust survivors to tell their stories to the general public. These include names such as Sally Perel, Esther Bejerano († 2021), Johanna Engel, Margit Meissner, Simone Polak, Eva Fahidi and many others.

Against the background that every generation brings with it its own peculiarity and therefore a cultural change is the result, that there will be a time without Holocaust survivors and that history can repeat itself and people can therefore fall into barbarism, forms and the diversity of reporting to the Shoah must always be maintained. In addition to the written canon such as German-Jewish literature, German-Jewish studies, German-Jewish remembrance culture and (cultural) history, oral traditions from contemporary witnesses must be conveyed to the recipients by means of advancing technology.

An attempt is made to present the ARD and ZDF media archive, in which a contribution is made to German-Jewish reporting. There are of course other channels, newspapers and magazines that are also actively involved in their online portal. However, since it is almost impossible to present all of them in one frame, the selection consists primarily of only the first two German television channels. After a short presentation of the media library, the contribution from the future podcast of the Tagesschau mal angenommen. Keine Holocaust-Zeitzeugen mehr? Was dann? will be demonstrated.

In the future podcast presented here, essentially two projects and, consequently, the implementation in school lessons are presented. The first project presented here is the so-called digital testimonies, which is a concept of the Ludwig Maximilians University in Munich and the Leibniz Data Center. The second project is the Anne Frank Learning Laboratory in Frankfurt am Main, which aims to sensitize the students to prejudices, stereotypes and the other.

The starting point of the whole project is namely the fear that on the one hand memories of the Holocaust could be lost and on the other hand there is a risk that if there are still efforts to preserve the memories, the emotional connection is not as strong as with a real one Holocaust witnesses when they tell their own story. The latter would be the story from the first source and the narrator reporting the personified story himself.

It is therefore intended to strive for an active prevention through knowledge against implicit and explicit secretion. Both terms, their application and function are then examined in more detail in the following chapters. In conclusion, it can be said that all efforts and endeavors have the goal of erecting a psychological memorial so that the atrocities are not forgotten and 
Auschwitz does not repeat itself. The act of not forgetting and thus, the act of remembering is considered to be the highest commandment. 


\author{
Einmal wird dieser \\ schreckliche Krieg doch \\ aufhören, einmal werden wir \\ auch wieder Menschen und \\ nicht allein Juden sein. \\ Anne Frank
}

\section{Einleitung}

Annelies Marie Frank (12.06.1929-1947 im KZ Bergen-Belsen) ist ein junges Mädchen aus Frankfurt am Main, der in der deutsch-jüdischen Literatur und in der europäischen Erinnerungskultur eine signifikante Funktion zugeschrieben wird. Ihre Feder ist deshalb so wichtig, da sie aus der Perspektive eines Kindes in das Grauen blickt und ihre Erfahrungen, Ängste, Wünsche und Vorstellungen für die Zukunft, immer wieder in Anbetracht der Naivität und Reinheit eines Kindes, wiedergibt. Ihr Tagebuch zählt in der deutsch-jüdischen Literatur des 20. Jahrhunderts zum Literaturkanon, die eine Konfrontation mit der kollektiven Schuld der nationalsozialistischen Zeit und eine Sicht in das Grauen der nationalsozialistischen Diktatur ermöglicht.

Neben dieser Art der schriftlichen Erinnerungsform, die einen wichtigen Platz in der Erinnerungskultur einnimmt, gibt es zudem noch mündliche Überlieferungen, die ebenso die gleiche Wertstellung haben (sollten). Dabei handelt es sich zumeist um Individuen, die das Grauen überlebt haben und deshalb auch als Holocaust-Überlebende bezeichnet werden. Diese wiederum leisten ihren Beitrag in der Sensibilisierung der Menschen auf allen sozialen, gesellschaftlichen und politischen Ebenen, sodass - um es mit den Worten Adornos auszudrücken - sich die „Barbarei“ nicht wiederholt. Vor allem öffentlich-rechtliche Kanäle wie etwa ARD und ZDF in erster Linie aber auch Zeitungen und Zeitschriften tragen primär zu den Sensibilisierungsbestrebungen der Gesellschaft bei und lassen Holocaust-Überlebende ihre Geschichte der ganzen Öffentlichkeit erzählen. Dazu zählen Namen wie etwa Sally Perel, Esther Bejerano († 2021), Johanna Engel, Margit Meissner, Simone Polak, Eva Fahidi und viele andere.

Vor dem Hintergrund, dass jede Generation ihre Eigenart mit sich bringt und deshalb ein kultureller Wandel die Folge ist, dass es einen Zeitpunkt ohne HolocaustÜberlebende geben wird, dass sich die Geschichte wiederholen kann und die Menschen aus dem Grund in die Barbarei fallen können, müssen Formen und die Vielfältigkeit der Berichterstattung an die Shoah stets aufrechterhalten werden. Neben dem schriftlichen Kanon wie etwa die deutsch-jüdische Literatur, deutsch-jüdische Studien, die deutschjüdische Erinnerungskultur und (Kultur-)geschichte müssen mündliche Überlieferungen der Zeitzeugen an die Rezipienten mittels der fortschreitenden Technologie übermittelt werden.

Es wird deshalb der Versuch unternommen das ARD und ZDF Medienarchiv vorzustellen, in der ein Beitrag zur deutsch-jüdischen Berichterstattung geleistet wird. Selbstverständlich gibt es weitere Kanäle, Zeitungen und Zeitschriften, die in ihrem Online-Portal ebenfalls aktiv mitwirken. Da es allerdings nahezu unmöglich ist, alle in einem Rahmen darzustellen, besteht die Auswahl in erster Linie nur aus den zwei ersten 
deutschen Fernsehkanälen. Nach einer kurzen Vorstellung der Mediathek, wird aus dem Zukunfts-Podcast der Tagesschau mal angenommen der Beitrag Keine HolocaustZeitzeugen mehr? Was dann? vorgeführt.

Es wird somit beabsichtigt eine aktive Prävention durch Wissen gegen implizite und explizite Ausgrenzung anzustreben. Beide Begrifflichkeiten der Vorbeugung und Aus- und Abgrenzung, ihre Anwendung und Funktion werden sodann in den folgenden Kapiteln näher beleuchtet.

\section{Kultureller Wandel}

In der Anthropologie wird die Terminologie Kulturwandel bevorzugt verwendet. Weitere Begrifflichkeiten, die in Form eines Synonyms angewandt werden, sind sozialer Wandel oder auch Umbruch. Unter dem Begriff des kulturellen Wandels wird zumeist die Entwicklung von sozialen Normen, Traditionen und Habitus verstanden, die dann eine Modifizierung eines alltags- und kulturbezogenen Verhaltens auslösen. Im Vordergrund stehen dabei jegliche Arten von beobachtbaren kulturellen Veränderungen. Exempli Causa sind es Ideen und Weltanschauungen der Menschen (s. Fuchs-Heinritz 1981). Eine gesonderte Stelle nimmt allerdings der kulturelle bzw. soziale Wandel ein, wenn es zu einer Änderung in Form einer Revolution zustande kommt (vgl. Boudon 1979: Kap. V, $\mathrm{VI})$.

Darüber hinaus konkurriert die Terminologie mit weiteren Begrifflichkeiten wie etwa Entwicklung, Fortschritt und Modernisierung (vgl. Immerfall 1991: 36). Dennoch sind die Gründe für einen kulturellen und sozialen Wandel nicht eindeutig darzulegen. Es sind meist mehrere Paradigmen betroffen, die einen Wandel auslösen. Somit ist eine Monokausalität für einen Wandel in diesem Sinne heutzutage ausgeschlossen. Deshalb kann man einen Wandel nicht auf einen bestimmten (Ur-)Kasus wie beispielsweise technische Innovationen, religiöse Einstellungen oder die Kultur zurückführen, da es weitreichende Interdependenzen gibt (vgl. Groh 2004: 13-31).

Generationen aber auch Generationskonflikte tragen eine besondere Funktion im Prozess des kulturellen Wandels bei, da der kulturelle Wandel meist mit Meinungsunterschieden verbunden ist. Nach Erich Fromm tendieren jüngere Generationen zum Beispiel meist dazu, aktiv etwas Anderes ausdrücken zu wollen, da sie der Ansicht sind, dadurch etwas bewirken zu können. Laut Fromm (vgl. 1977: 184) sei es ein genetisch begründetes Grundbedürfnis eines Einzelnen das aktive Handeln auszulösen, die im Anschluss die eigentliche Antriebskraft für jeglichen Wandel ausmacht. Es sei somit ein Ausdruck des freien Willens. Auch Jan Assmann fügt sich jenem Gedankengang hinzu und setzt fort, dass es meist Jüngere sind, die im Lösungsprozess eines Problems von ihrer älteren Generation eine Veränderung erwarten. Somit sind Individuen aus unterschiedlichen Generationen Träger und Akteur eines sozialen Wandels (vgl. Assmann 2013). Wenn es zu einem erheblichen Meinungsunterschied innerhalb der unterschiedlichen Generationen kommt, die Weltund Menschenbilder sich stark voneinander differenzieren, so ist ein Wandel innerhalb 
der diversen gesellschaftlichen Ebenen umso beschleunigter die Folge (vgl. Lévi-Strauss 1975). Es sollte hier ebenfalls vermerkt sein, dass technologische Innovationen dazu beitragen, den Kulturwandel anzutreiben, wie es etwa heutzutage mit dem Internet und den sozialen Medien der Fall bei den Jugendlichen ist.

\section{Die Mediathek der ARD und ZDF}

Seit 2007 strahlen die Arbeitsgemeinschaft der öffentlich-rechtlichen Rundfunkanstalten der Bundesrepublik Deutschland (ARD) und das Zweite Deutsche Fernsehen (ZDF) das sogenannte Video-On-Demand Angebot auf den jeweiligen Internetplattformen aus. Das Video-On-Demand ist ein Angebot, in der man sich auf Anforderung Videos online anschauen (streamen) oder direkt herunterladen (downloaden) kann. Dabei gibt es verschiedene Rubriken, die in den Online-Plattformen aufgelistet sind.

Die ARD bietet beispielsweise ein breites Spektrum an Kategorien von Abrufvideos an. Diese lassen sich unterteilen in die Nachrichtensendung wie etwa die Tagesschau, politische Sendungen wie Report Mainz oder Report München, Fernsehreihen oder -sendungen wie Tatort oder Lindenstraße, Kultursendungen wie Druckfisch oder ttt - titel, thesen, temperamente, Kinderprogramme wie Die Sendung mit der Maus, aber auch wissenschaftliche Sendungen wie $W$ wie Wissen oder Wissen vor acht.

Das ZDF hingegen ist hauptsächlich in fünf Kategorien unterteilt. Diese sind Nachrichtenblöcke wie etwa heute-journal, politische Sendungen wie Frontal, Informationssendungen wie drehscheibe oder Volle Kanne, wissenschaftliche Reportagen wie Terra $X$ und ganze Fernsehreihen wie Herzensbrecher - Vater von vier Söhnen.

Neben den Videodigitalisaten gibt es auch eine Audiothek der ARD und ein Podcast-Angebote vom ZDF mit rund 25 Sendungen, die man herunterladen und auf einem iPad, iPod touch, iPhone und anderen vergleichbaren Geräten sich anhören kann. Wichtig für den folgenden Beitrag ist allerdings der Zukunftspodcast der ARD mal angenommen. Der Zukunftspodcast simuliert hier politische Ideen der Zukunft, wirft Zukunftsszenarien vor und diskutiert diese aus. Ziel der 30-minütigen Ausstrahlungen ist es, mögliche Szenarien vorauszudenken und ein Gedankenexperiment zu führen, in dem man dem Zuhörer nahelegt, was passieren könnte, wenn der diskutierte Diskurs zu einer Wirklichkeit wird.

In einem dieser Zukunftspodcast wird beispielsweise die Frage ausdiskutiert Keine Holocaust-Zeitzeugen mehr? Was dann? Neue Ansätze der Erinnerung. Da nun seit dem Zweiten Weltkrieg, und diesbezüglich auch dem Holocaust, genau 76 Jahre vergangen sind, rückt die Realität näher, dass es bald keine Holocaust-Zeitzeugen mehr geben wird. Wer wird dann die Aufklärungsarbeiten leisten, erinnert sich eine Kultur bzw. die Folgegeneration weiterhin an die Gräueltaten oder geraten die Erinnerungen in Vergessenheit? Aber auch wenn man sich an die Shoah erinnert, so sind beispielsweise private Auftritte, institutionelle Besuche und Aufklärungsarbeiten der Zeitzeugen umso 
seriöser, rührender, packender, beeindruckender und der emotionale Bezug zu den Rezipienten höher. Die Folgen sind umso gelungener, erfolgreicher und produktiver. Demzufolge versucht man mithilfe neuester Technologien den herrschenden emotionalen Bezug vor allem zu Kindern und Jugendlichen aufrechtzuerhalten und weiterhin zu intensivieren. Im Zeitalter des Kulturwandels, in der Jugendliche ein größeres Interesse an Smartphones und den sozialen Medien wie Youtube, Facebook, Instagram, Snapchat oder Twitter aufzeigen, versucht man, auch mithilfe der technologischen und (sozial)medialen Kanäle die Rezipienten zu erreichen. Deshalb sollte es nicht wunderlich sein, wenn Holocaust-Zeitzeugen in einem kleinen Interview und in Video-Format aufgenommen werden. Diese Unterhaltung ist zielgerichtet und dient zum größten Teil pädagogischen Zwecken. Demnach tauchen Holocaust-Zeitzeugen in Youtube-Kanälen auf, die von allen anderen sozialen Medien ebenfalls abrufbar sind. Diese können dann von Kindern und Jugendlichen, aber auch von Erwachsenen zu jeder Zeit und zu jedem Ort angehört und/ oder angeschaut werden. Allein wenn man in der ARD-VideoMediathek den Begriff Holocaust in die Suchleiste eingibt, so werden 186 Videos in Bezug auf die Shoah aufgelistet, in der zum größten Teil Holocaust-Zeitzeugen auftreten und ihren Beitrag zur Sensibilisierung und Aufklärung der Gesellschaft leisten. In der ZDF-Mediathek hingegen ist die Anzahl der Videos umso höher und beträgt nach Angaben der offiziellen Webseite mehr als 100 Treffer. Darüber hinaus bietet das ZDF eine Dokumentationsserie über den Holocaust, die man sich auch online ansehen (streamen) kann. Die Serie Das Deutsche Reich im freien Fall setzt sich mit der Frage auseinander, wie aus einer krisenhaften Demokratie eine Apokalypse mit Krieg und Völkermord entstehen konnte. Hier werden der Aufstieg und der Untergang des Nationalsozialismus dargestellt.

\section{Zukunftspodcast mal angenommen - Keine Zeitzeugen mehr? Was dann?}

In dem hier vorgestellten Zukunftspodcast werden wesentlich zwei Projekte und infolgedessen auch die Implementierung im Schulunterricht vorgestellt. Das erste hier präsentierte Projekt ist das sogenannte Digitale Zeugnis, welches ein Konzept der Ludwig-Maximilians-Universität in München und dem Rechenzentrum Leibniz ist. Das zweite Projekt ist das Anne Frank Lernlabor in Frankfurt am Main, in der eine Sensibilisierung der Schüler und Schülerinnen bezüglich der Vorurteile, den Stereotypisierungen und dem Anderem erstrebt wird. 


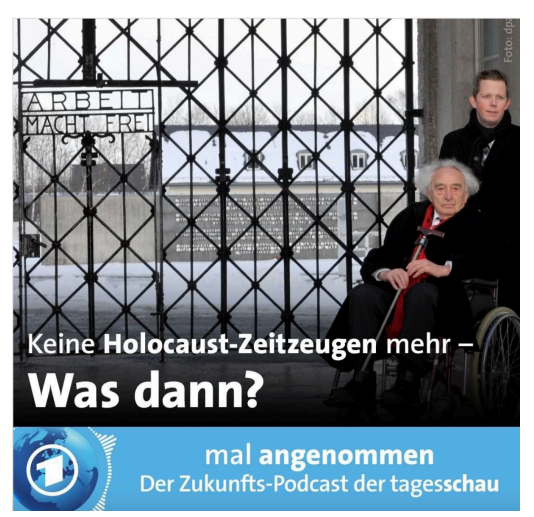

Abb. 1: ARD Zukunftspodcast über die Holocaust-Zeitzeugen

Ausgangspunkt des ganzen Vorhabens ist nämlich die Befürchtung, dass einerseits die Erinnerung an den Holocaust verloren gehen könnte und andererseits die Gefahr besteht, dass, wenn es immerhin noch Bestrebungen der Aufrechterhaltung an die Erinnerungen gibt, der emotionale Bezug nicht so stark ist wie bei einem realen Holocaust-Zeitzeugen, wenn diese ihre eigene Geschichte erzählen. Letztere wäre nämlich Geschichte aus erster Quelle und der/ die berichtende Erzähler/in - also ein Holocaust-Zeitzeuge - die personifizierte Geschichte höchstpersönlich.

Jan Assmann, ein Pionier der Gedächtnisforschung, gibt zu erkennen, dass es zwei wesentliche Formen des Gedächtnisses gibt, die die Erinnerungen aufrechterhalten. Zum einen prägt er den Begriff des „kommunikativen Gedächtnisses“ und zum anderen des „kulturellen Gedächtnisses“. Wenn also Holocaust-Zeitzeugen ihre Geschichte nicht mehr weitererzählen können, so gibt es keinen Austausch mehr zwischen Sprecher und Rezipient. Die Folge ist der Untergang des kommunikativen Gedächtnisses, denn wenn die Träger der Kommunikation aufhören zu existieren, so gibt es auch das kommunikative Gedächtnis nicht. Das kulturelle Gedächtnis hingegen sei unabhängig von ihren Trägern und zeitlich betrachtet nicht an die Dimensionen gebunden (Assmann 2013: 56). Sie ist zeitlos, hat spezialisierte Traditionsträger und ihre Formen der Tradierung zeichnen sich charakteristisch durch einen hohen Grad an Geformtheit und einer zeremoniellen Kommunikation aus. Der tradierte Sinn weist eine Außeralltäglichkeit auf. Demzufolge ergibt sich die Frage, wie der emotionale Bezug primär zu Kindern und Jugendlichen und sekundär zu den Erwachsenen erstellt und intensiviert werden kann.

\section{Das digitale Zeugnis}

Lernen mit digitalen Zeugnissen ist ein Projekt der Ludwig-Maximilians-Universität München und dem Rechenzentrum Leibniz. In dem Pilotprojekt geht es um eine dauerhafte Herstellung zu den Erinnerungen an den Holocaust. Mit einfachen Worten werden Holocaust-Zeitzeugen interviewt, aufgenommen und die Aufzeichnungen verund bearbeitet. Mithilfe neuester Technologie und innovativer Forschung sollen sodann die Ausstrahlung der drei-dimensionalen Aufnahmen dafür sorgen, dass Rezipienten oder Zuschauer als auch Zuhörer eine Art Kommunikation mit dem virtuellen Zeitzeugen 
aufbauen können. Demnach hat man die Möglichkeit Fragen an die HolocaustÜberlebenden über den Holocaust und die diesbezüglichen Erfahrungen zu stellen, die dann im Nachhinein von dem virtuellen Zeitzeugen beantwortet werden. Das Resultat ist ein komplexer Produktions- und Interaktionsprozess, wodurch intendiert wird, eine Vermittlungsaufarbeitung durchzuführen.

Diese Zeugnisse der Medienprodukte bieten neue Perspektiven an, wie eine interund transdisziplinäre Beschäftigung rund um das Thema des Holocaust ${ }^{6}$ und der nationalsozialistischen Diktatur stattfinden können (vgl. Ballis und Gloe 2019; 2020). Die in 3D-Format aufgezeichneten Digitalisate werden beispielsweise seit $2015 \mathrm{im}$ englischsprachigen Raum, primär in den Vereinigten Staaten und das Vereinigte Königreich, der Öffentlichkeit zugänglich gemacht. Dafür tragen Holocaust-Museen in den jeweiligen Ländern ihren Beitrag bei. Die Zeitzeugen erzählen hier nicht nur ihre eigene Geschichte, sondern man hat sogar die Möglichkeit bis zu rund 2.000 Fragen über ihr Leben und den eigenen Erfahrungen zu stellen, die dann virtuell beantwortet werden.

Die volumetrisch oder stereoskopisch gefilmten Aufzeichnungen sollen eine möglichst genaue Visualisierung des Lebens von Überlebenden und in ihrer Lebensgröße darstellen. Dabei spielt die Wechselreaktion von Frage-Antwort den innovativen Kern der Digitalisate. Hier werden mündlich gestellte Fragen in das Format eines Textes transformiert, die dann im Anschluss an einen Rechner der Sprachverarbeitung übermittelt werden. In der medialen Datenbank des Speichers werden sodann nach passenden Antworten gesucht. Daraufhin wird der Text wieder in Laute transformiert und der virtuelle Zeitzeuge bzw. das digitale interaktive 3D-Zeugnis gibt die Antwort. Zwei grundlegende Schritte sind hier demnach zu unterscheiden. Zum einen ist es der Akt der Wahrnehmung und zum anderen der Akt der Produktion, mit anderen Worten dem In-Put und dem Out-Put (vgl. Elleström 2018: 286). „The act of production may thus be simple and direct, as well as complex and indirect. It may furthermore include stages of storage“ (ebd. 2018: 283). Für die in deutscher Sprache erstmals aufgenommenen und zum Zwecke der Erinnerungsrekonstruktion durchgeführten Zeugnisse werden verschiedene Kommunikationsmodelle näher unter die Lupe genommen. Anja Ballis, Florian Duda und Markus Gloe beginnen mit den Modellen von Shannon und Weaver (1948) bis hin zu Stuart Hall (1980) und versuchen sodann die theoretische Explanation für die 3DZeugnisse zu vermitteln. Demnach lässt sich folgendes Schema eines medienzentrierten Kommunikationsmodelles herauskristallisieren: 


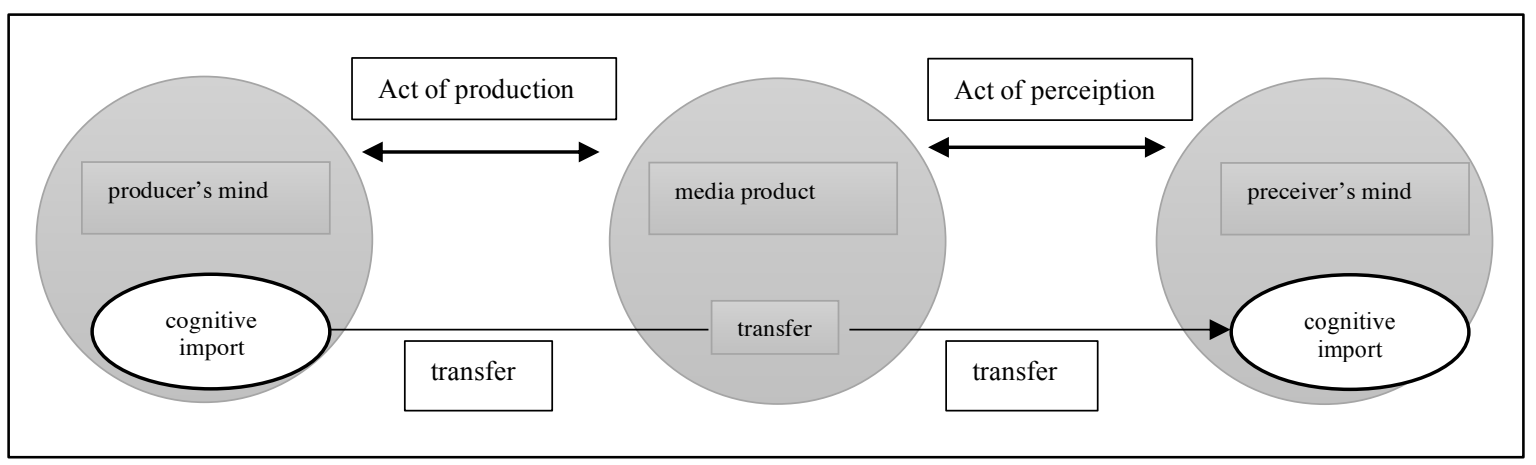

Abb. 2: Kommunikationsmodell (Elleström 2018: 282)

Für das geeignetste Modell wird sodann eine Synthese zwischen dem medienzentrischen Modell von Elleström und weiteren Modellen, wie etwa die von Reichertz (vgl. 2009: 113), durchgeführt. In dem Mensch-Maschine Modell gibt es eine „one-to-one“ Kommunikation, d. h. beispielsweise, dass Rezipienten Fragen an den virtuellen Zeitzeugen stellen, und eine ,one-to-many“ Kommunikation, in der der Zeitzeuge an eine Mehrzahl von Menschen antwortet. Demzufolge entsteht in Anlehnung an das Kommunikationsmodell von Ellenström folgendes und für das 3D-Zeugnis passende Modell:

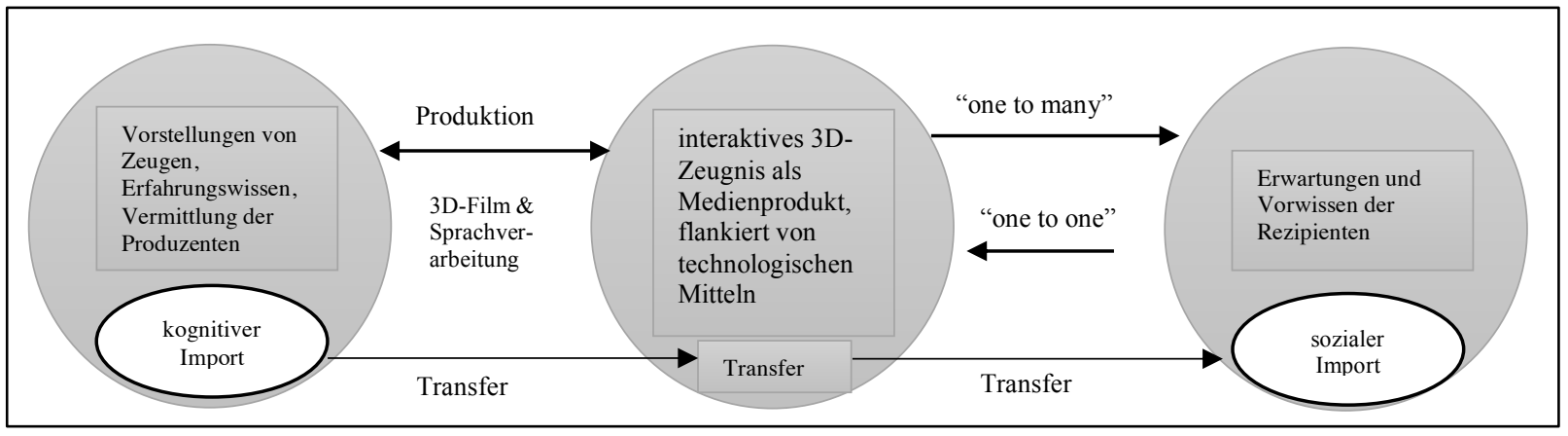

Abb. 3: An 3D-Zeugnisse angepasstes Kommunikationsmodell in Anlehnung an Elleström (Ballis 2021: 10)

Die immer voranschreitende Technologie mit der innovativen Forschung bietet eine Ansammlung und Mehrperspektivität in puncto der digitalen Zeugnisse dar. Jene ermöglichen in der Folge das digitale Erinnern. Diese vielfache Anschlussmöglichkeit der jeweiligen Rezipienten mit ihren Erwartungen, dem Vorwissen, den persönlichen, ideologischen und politischen Überzeugungen ist eine Reaktion des verbalisierten Wissens (vgl. Krämer 2008: 229), welches von einem virtuellen Zeitzeugen ausgestrahlt wird (vgl. Ballis 2020: 239). Der Transfer besteht nicht nur aus den übermittelten subjektiven Erfahrungen und Erlebnissen eines Mediums, sondern aus ganzen Formen der verbalen und nonverbalen Kommunikation; darunter der Körpersprache, Mimik, 
Gestik, Prosodie und weitere. Die Vermittlung der Emotionen von einem virtuellen Zeitzeugen ist demnach durchaus hoch.

Im Gegensatz zu dem bisher Geschilderten ist Kritik nicht auszuschließen, denn die digitalen Zeugnisse sind vorprogrammiert und ersetzen nicht die Erlebnisse. Es können exempli causa Fragen gestellt werden, die einerseits vorher nicht programmiert worden sind oder andererseits, ähnelnde Fragen, die bereits in der Datenbank abgespeichert sind, die wiederum eine Software nicht klar zu unterscheiden weiß, weshalb es beispielsweise zu Verwirrungen kommen kann. Zum Beispiel könnte die Frage gestellt sein „Habt Ihr überlebt?““. Auf der Suche nach der Antwort in der Datenbank kann es zu Verwirrungen kommen, wenn es ähnliche Fragen im Korpus gibt wie etwa „Hat Ihr Vater überlebt?“ oder „Hat Ihr Großvater überlebt?“. Da das System an jener Stelle aufgrund statistischer Daten eine Antwort sich aussucht, kann in der Konstruktion der Antworten ein Umstand entstehen, in der der Wahrheitswert der Aussage niedrig bzw. dann auch nichtzutreffend ist (vgl. Ballis 2021: 18f.).

\section{Implementierung im Deutschunterricht}

Der Einsatz der digitalen Zeugnisse in Schulen und weiteren institutionellen Stätten trägt eine besondere Funktion und Signifikanz. Auf diese Weise ist es nämlich in einem Zeitalter ohne Holocaust-Zeitzeugen auch noch möglich, an die Gräueltaten weiterhin sich zu erinnern, einen starken emotionalen Bezug zu den Rezipienten herzustellen und den Menschen sowohl rational als auch emotional aufzuzeigen, was für Folgen Ideologien der Extreme jeglicher Art einnehmen können. Die Tradierung stereotyper Bilder und die Rekapitulation der Geschichte sollen Erinnerungen wachhalten und ein Mahnmal gegen das Vergessen setzen. ${ }^{1}$ Die Intention liegt hierbei in der Erziehung und Sensibilisierung ganzer Kollektive.

Nach Adorno sollte diese Erziehung bereits im Kindes- und jugendlichen Alter anfangen. Deshalb ist die Implementierung der Digitalisate essentiell, da die an die Zeitzeugen gerichteten Fragen äußert bedacht aus einem reichlichen Fragenkatalog durch eine Kommission ausgewählt worden sind. ${ }^{2}$ In zwei solcher digitalen Zeugnisse, von Abba Noar und Eva Umlauf, wurden zum Beispiel zehn Fragen mit dem höchsten Ranking ausgewählt, die dann an die Holocaust-Zeitzeugen gestellt wurden. Die

\footnotetext{
${ }^{1}$ In der Literatur beschäftigen sich deutschsprachig-jüdische Autoren auch mit dem Akt des Erinnerns und setzen ein Mahnmal gegen das Vergessen. Siehe hierzu: Coşan, Leyla und Habib Tekin (2021): Stefan Zweigs Werke im Lichte der jüdischen Erinnerungskultur. In: Coşan, Leyla und Mehmet Tahir Öncü (Hrsg.) (2021): Jüdische Lebenswelten im Diskurs. Berlin: Logos. S. 125-145.; Coşan, Leyla und Habib Tekin (2021): Bilder der Judenfeindschaft in den Werken von Jakob Wassermann. In: Diyalog. Interkulturelle Zeitschrift für Germanistik. 2021/1. S. 3-22.; Tekin, Habib (2021): Bilder der Judenfeindschaft in den Werken von Jakob Wassermann. Berlin: Logos; Demirkıvıran Sine (2021): Mirjam Pressler „Nathan und seine Kinder“: Adaptation und Rewriting für junge Leser. In: Coşan, Leyla und Mehmet Tahir Öncü (Hrsg.) (2021): Jüdische Lebenswelten im Diskurs. Berlin: Logos. S. 223-247.

${ }^{2} \mathrm{Zu}$ der vollständigen Komissionsliste siehe: Schwendemann, Lisa und Ernst Hütl (2021): 1000 Fragen an Abba Naor und Eva Umlauf - Die Rolle der Fragen und Antworten für die Erstellung interaktiver 3DZeugnisse. In: Ballis, Anja et al. (Hrsg) (2021): Interaktive 3D-Zeugnisse von Holocaust-Überlebenden. Chancen und Grenzen einer innovativen Technologie. Eckert. Dossiers 1 (2021). S. 29.
} 
Aufzeichnungen und der spätere Einsatz sollten zum Nachdenken anregen. Die zehn Fragen an Abba Noar lauten:

1. Was ist die wichtigste Botschaft, die Sie übermitteln wollten?

2. Wie fanden Sie heraus, was mit Ihrer Familie geschehen war?

3. Warum geben Sie Ihr Zeugnis weiter?

4. Haben Sie nach dem Holocaust Antisemitismus erlebt?

5. Was würden Sie einem Holocaustleugner entgegnen?

6. Wie haben Sie herausgefunden, dass Ihr älterer Bruder erschossen worden war?

7. Wie haben Ihre Eltern reagiert, als Ihr älterer Bruder erschossen worden war?

8. Wie konnten Sie das Lager Stutthof überleben?

9. Was passierte mit Ihrem Vater während des Holocaust?

10. Was waren die letzten Worte, die Ihre Mutter Ihnen sagte?

Die speziell ausgewählten Fragen, die an Eva Umlauf gestellt wurden, lauten wie folgt:

1. Was ist die wichtigste Botschaft, die Sie übermitteln wollen? Warum haben Sie Ihr Tattoo behalten?

2. Welche Erinnerungen haben Sie an Ihren Vater?

3. Wann fragten Sie das erste Mal nach der Bedeutung Ihrer eintätowierten Nummer?

4. Wo liegt für Sie Ihre Heimat heute?

5. Sahen Sie sich in der Zeit nach Ihrem Umzug nach München antisemitischen Haltungen oder Handlungen ausgesetzt?

6. Wie beeinflusst Ihre Vergangenheit Ihre Arbeit?

7. Würden Sie uns Ihr Tattoo zeigen?

8. Welcher Nationalität fühlen Sie sich heute zugehörig?

9. Was erzählte Ihre Mutter von Ihrem Vater? (Schwendemann / Hütl 2021: 31).

Das Lernen mit digitalen Zeugnissen wird vom Ministerium für Bildung und Forschung unterstützt. Es war eigentlich geplant, dass man die Zeugnisse vor allem in bayrischen Schulen ab dem Frühjahr 2020 einsetzte. Aufgrund der Corona-Pandemie war dies allerdings nicht möglich, weshalb es zu einer Verzögerung im Einsatz an Schulen gekommen ist (vgl. Gloe 2020).

Anja Ballis ${ }^{3}$, eine Germanistikprofessorin an der Ludwig-MaximiliansUniversität, untersucht Möglichkeiten, wie interaktive 3D-Zeugnisse in Deutschlehrer und -lehrerinnenbildung integriert werden können (vgl. Ballis 2021). In ihrem Aufsatz

\footnotetext{
${ }^{3}$ Florian Duda (s. 2021: 43-62) beschreibt in seinem Aufsatz die Funktionsweise der Sprachverarbeitung, Anja Ballis (s. 2021: 83-110) geht auf die Wirkung des Projektes und Implementierung im Deutschunterricht ein, Daniel Kolb (s. 2021: 63-82) hebt die technischen Aspekte der Anwendung hervor, Fabian Heindl (s. 2021: 111-129) beschreibt den Produktionsprozess von digitalen Zeugnissen und Markus Gloe (s. 2021: 130-146) erläutert in seiner Fallstudie die Präsentation der Zeugnisse in Holocaust Museen in den Vereinigten Staaten und dem europäischen Kontinent.
} 
lenkt sie auf die Wichtigkeit des Einsatzes der Digitalisate in Schulen ein und nennt die Verwendungsmöglichkeiten. Lehramtsstudierende seien

\begin{abstract}
vorzubereiten auf neue Kommunikations- und Arbeitsformen, die durch den ständigen Zugriff auf Wissen geprägt sind. Zudem sollen sie befähigt werden, ,selbstständig mit neuen Techniken umzugehen, diese sinnvoll einzusetzen und kritisch zu reflektieren`.
\end{abstract}

(KMK-Strategie 2016: 42)

Bei Ballis handelt sich im Wesentlichen um das Imperativ „Nie wieder“" oder mit Worten Adornos, „dass Auschwitz sich nicht wiederhole.“ Sie hebt die Relevanz des Einsatzes der Zeugnisse in der Form hervor, dass Lehramtsstudierende ein kritisches Geschichtsbewusstsein aufbauen müssen. Dies geschehe, wenn man Trauer und Mitleid für unschuldige Menschen empfindet, aber auch zugleich durch „Wut und Verzweiflung angesichts der Verbrechen an unschuldige Menschen“" (Ballis 2021: 93). Sie vermerkt ebenfalls, dass solche Gefühle zwar meist als obligatorisch angesehen sind, jedoch nicht immer so empfunden werden (vgl. Krieg 2015: 124, zit. n. Ballis 2021: 93).

Letztendlich soll an dieser Stelle die personale fachliche Bildung nach Ballis (2021: 104) ohne weitere Erklärungen, dargestellt werden, um auf die Notwendigkeit zu verweisen, wieso es wichtig ist, die digitalen Zeugnisse in den Schulunterricht einzusetzen:

1. Empathie mit den Opfern von Gewalt und Terror, mit und ohne Bezug zum familiären Gedächtnis der Schüler*innen, empfinden,

2. Wissen aus biografischen Erzählungen unter Berücksichtigung ihrer Medialität erschließen,

3. den „Augenblick“, das „Faszinosum“ als Kategorie der Bildung erleben,

4. Emotionen im Umgang mit Zeugnissen von Überlebenden des Holocaust reflektieren,

5. Lehren aus der Vergangenheit für die eigene Gegenwart ziehen,

6. Chancen und Gefahren der Digitalisierung an einem historischen Beispiel erfassen und durchdenken.

\title{
Anne Frank Lernlabor in Frankfurt am Main
}

Das Bundesprogramm Demokratie leben! fördert deutschlandweit Projekte auf Bundes-, Landes- und Kommunalebene. Mehr als 100 Modellprojekte entwickeln und erproben innovative Ansätze, die die Demokratieförderung zur Aufgabe machen, darunter auch die Bildungsstätte Anne Frank in Frankfurt am Main. Beispielsweise beschäftigt sich die Stätte mit politischer Bildungsarbeit wie etwa den aktuellen Formen des Antisemitismus und entwickelt ein neues Format, um gegen jegliche Formen der Diskriminierung entgegenzuwirken. Es ist zum Ziel gemacht worden, Jugendliche und Erwachsene für die aktive Teilnahme an der Stärkung der Demokratie zu motivieren, wobei zugleich ein gesunder Umgang mit menschenfeindlichen Ideologien trainiert wird. Jegliche Formen von Gewalt, Rassismus und Diskriminierung sind relevante Begrifflichkeiten, mit der sich die Stätte auseinandersetzt und Aufklärungsarbeiten zu leisten versucht, denn im Zuge der Globalisierung, der ansteigenden Migration(sbestrebung), der immer 
zunehmenden Polarisierung innerhalb der Gesellschaft, lassen sich Anlässe für Diskriminierungen, oder sogar der Überschneidung von verschiedenen Formen der Diskriminierung - auch genannt als die Intersektionalität -, gegenüber einer Person nachweisen. Demzufolge gibt es im Lernlabor drei wesentliche Themenblöcke: (1) Diskriminierung, (2) Normalität und (3) Demokratie.

$\mathrm{Zu}$ den pädagogischen Grundlagen gehören Elemente der Konfliktpädagogik und die Ausbildung und Erziehung zur Demokratie und den Menschenrechten.

Durch die Darstellung verschiedener Perspektiven soll ein äußerer Konflikt in ein inneres

Dilemma umgewandelt werden. Dies kann Unbehagen, Erstaunen, Nachdenklichkeit und

Empathie hervorrufen und nach und nach auch zur Übernahme neuer Perspektiven bzw.

einem Umdenken in Bezug auf Diskriminierung führen. (Eckmann 2006: 210)

Die drei Themenpunkte werden durch verschiedene farbliche Markierung im Labor kenntlich gemacht, in der Museenbesucher die diversen Stationen zu durchlaufen haben. Die erste Anlaufstelle ist die orangene Station mit dem Motto „Mensch, du bist gleichwertig“. Dies ist der erste Themenblock, und zentrale Fragen und Überlegungen beschäftigen sich mit der Diskriminierung im Alltag. Die zweite und graue Station mit dem Motto „Mensch, du kannst sein wie du bist und sein willst!“ beschäftigt sich mit der Normalität, d. h. also wie der Begriff ,normal“ zu verstehen und, was „normal“ ist, wer die Grenzen zur Normalität setzt. Mit der letzten grünen Station versucht man Jugendliche und Erwachsene in das Demokratiewesen zu integrieren und anzuregen am demokratisch-gesellschaftlichen Leben aktiv mitzuwirken. Das Motto hierzu lautet „Mensch, du kannst (mit-)bestimmen!“

Im Folgenden sollen nun drei Beispiele in den vorgeführten Themenblöcken dargestellt werden, die das aktive Lernen im Lernlabor ermöglichen. In einer Station wird zum Beispiel der Körper eines Besuchers von einem Bodyscanner gescannt. Hier werden bestimmte Eigenschaften sinnlos den verschiedenen Körperteilen zugeordnet. Anschließend kommt es zu einer positiven und/oder negativen Bewertung, woraus eine Hierarchisierung zwischen Einzelnen und Gruppen entstehen. Die einer Person arbiträr zugeschriebenen Eigenschaften sollen zum Nachdenken anregen, wie es ist, nach dem äußeren Erscheinungsbild kategorisiert zu werden. Dadurch sollen Mechanismen der Diskriminierung aufgezeigt und Besucher diesbezüglich sensibilisiert werden. 


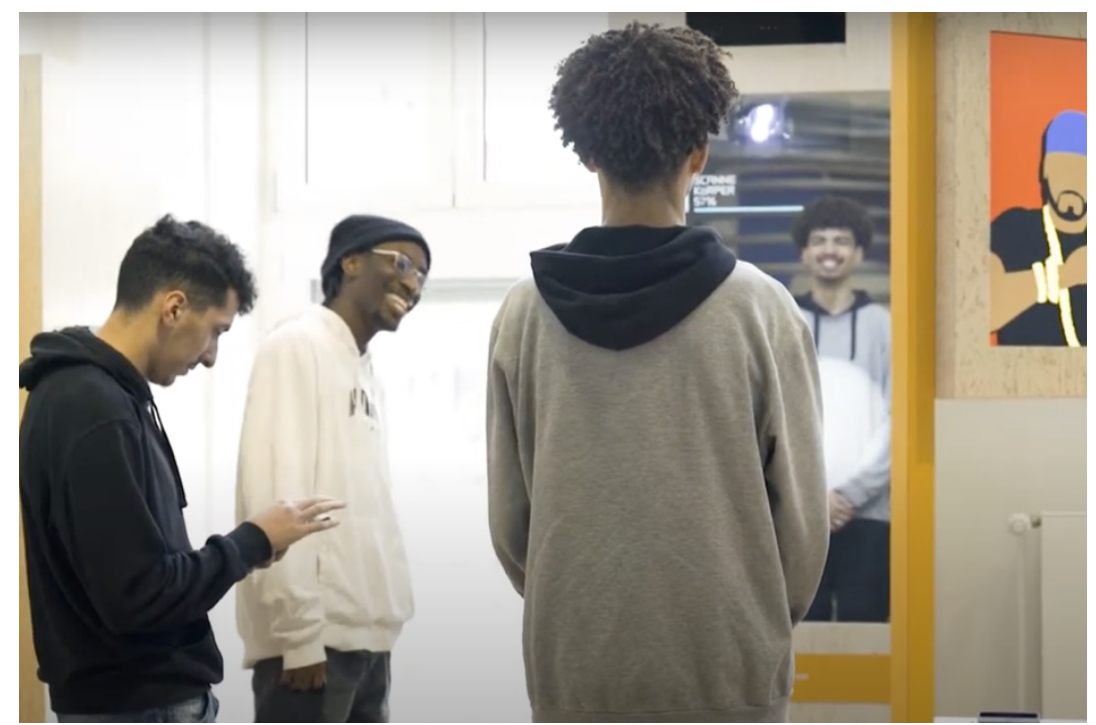

Abb. 4: Jugendlicher (rechts) vor einem Bodyscanner

In einer weiteren Station werden in einer Schublade Titelbilder von Illustrierten gezeigt, die eine satirische Art der diskriminierenden Praxis nachweisen.

Beispielsweise geht es um die Darstellung einer Familienkonstellation und die Rolle der Frau wie sie etwa in einer als ,normal“ eingestuften Familie aufgefasst werden: Es ist ein heterosexuelles Paar, sie haben Kinder und Haustiere. Der Vater als Familienoberhaupt geht arbeiten und die Mutter ist im Haushalt tätig. Diese klischeehaften Erscheinungsformen einer ,normalen“ Familie werden sodann an andere Familienformen wie etwa gleichgeschlechtliche Paare angewendet. Anschließend werden Jugendliche nach ihrer Wahrnehmung und Meinung gefragt, wodurch Bewertungen konstruiert werden wie etwa „nicht normal“ oder ,anders“. Infolgedessen sollen Jugendliche erkennen, wie breit das persönliche Repertoire an Vorurteilen und Voreinstellungen gegenüber dem Fremden ist. 


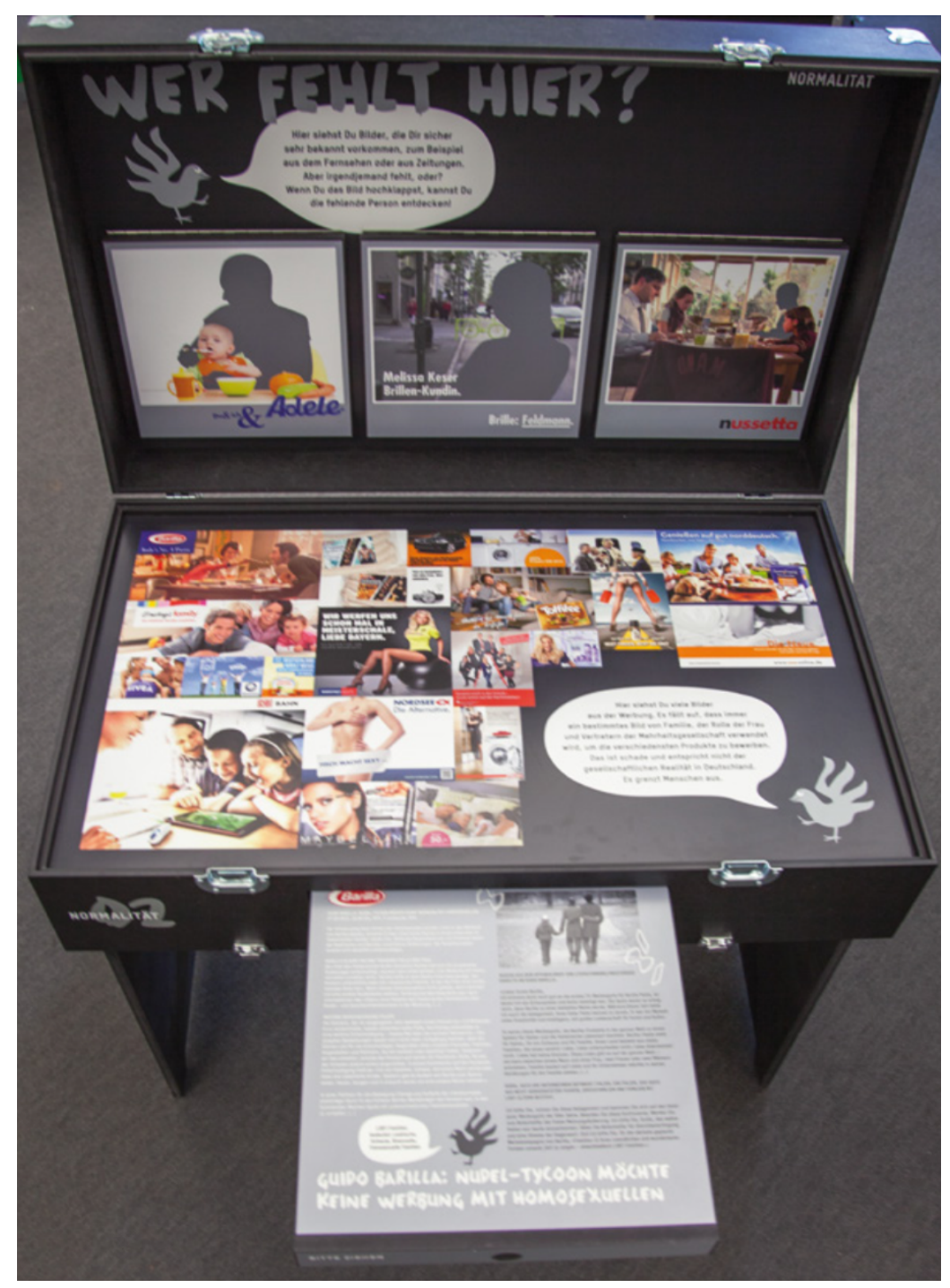

Abb. 5: Station Schublade mit Titelbildern

Das dritte Beispiel bringt eine kritische Auseinandersetzung mit medialer Berichterstattung und der Werbesprache zum Vorschein. Werbungen im Fernsehen oder im Internet, in Zeitschriften oder Zeitungen beeinträchtigen die Denkweise und Wahrnehmung der Einzelnen und ganzer Kollektive stark. Demnach werden verschiedene Werbungen aufgezeigt und die Frage in die Mitte des Diskurses gestellt: Inwieweit ist die Werbung als diskriminierend zu verstehen? In der Station geht es weniger um die Auseinandersetzung mit verschiedenen Marken oder Firmen und inwiefern sie Diskriminierung ausüben, sondern vielmehr um die Tatsache, dass (un)bewusst oder (un)bedacht diskriminierende Werbesprache vorzufinden ist und ebenso verwendet wird. Ob die Firmen oder Marken eine Diskriminierung ausüben, ist der Diskussion ausgeschlossen. Der Fokus liegt auf dem Akt der Wahrnehmung von verschiedenen Formen der Diskriminierung. 


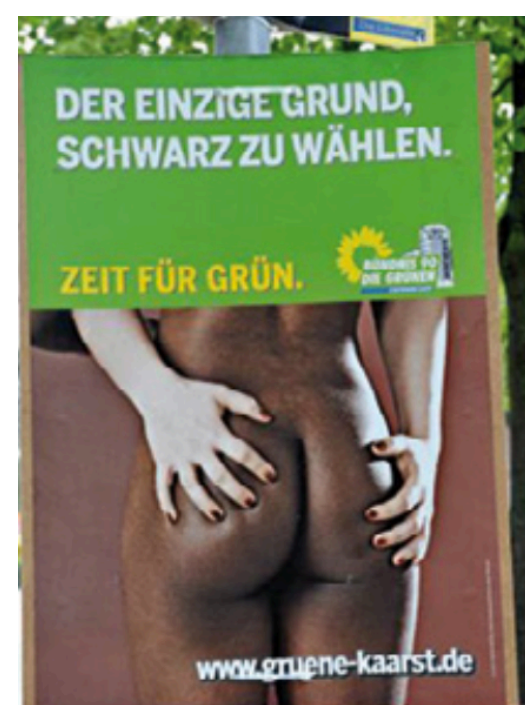

Abb. 6: Wahlplakat der Grünen

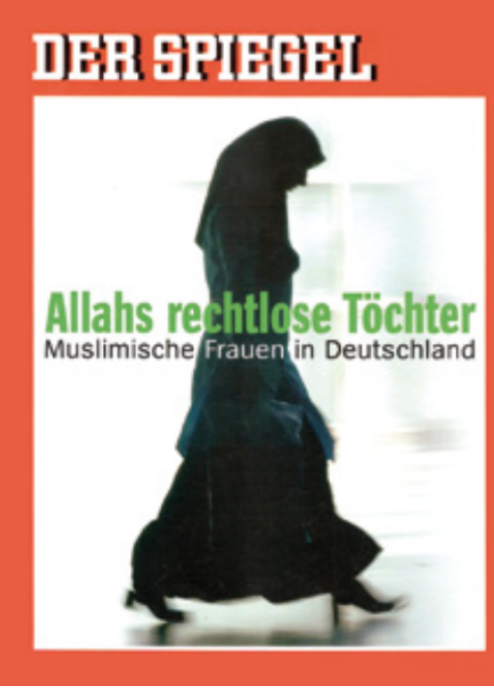

Abb. 7: Coverbild der Zeitschrift Der Spiegel

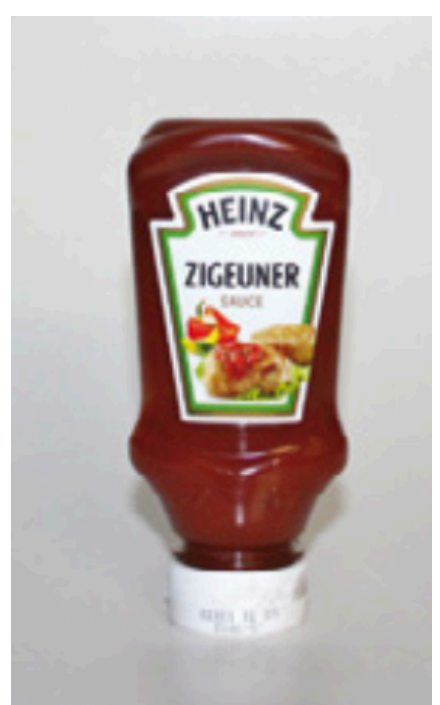

Abb. 8: Etikett der Heinz Ketchup

Hier in Abbildung 6, 7 und 8 sind verschiedene Formen von Werbesprache vorgeführt, die das Nachdenken und Hinterfragen anregen sollen, ob es diskriminierende Wirkungen hat.

Wenn eine Partei im Deutschen Bundestag für Gleichheit wirbt und dabei den Kontrast von schwarz-weiß mit einem sexistischen Plakat darstellt, so soll dies zum Nachdenken anregen, inwiefern hier Paradigmen der Diskriminierung - Ethnie und Sex(ualität) - eingebettet sind. Ebenso könnte man das provokative Coverbild von Der Spiegel analysieren, denn eigentlich wird in dem Beitrag eine Nachricht vermittelt, die auf abendländische Grund- und Menschenrechte verweist, wobei eine völlig verschleierte, potentiell muslimische Frau mit Stöckelschuhen auf dem Coverbild abgebildet ist, die ihre Blicke senkt. Sind es nun die Oppositionsachsen zwischen West vs. Ost, Frau vs. Mann, Orient vs. Okzident, abendländlich modern vs. morgenländlich konservativ, oder ist es die Tatsache, dass es sich hierbei um einen provokativen und verkaufsstrategischen Grund handelt. Jenes ist der Diskussion und dem weiteren Nachdenken offen. Im letzten Beispiel der Heinz-Ketchup Werbung wird sodann der semantisch abgewertete Begriff Zigeuner stellvertretend und stereotypisch für die Angehörige der Roma und Jenische verwendet.

Es wird demnach bezweckt, das am Ende der drei Stationen die Schülerinnen und Schüler des Lernlabors zu der Auffassung gelangen und sich dessen bewusst werden, wie stark sie selber der Diskriminierung ausgesetzt sind und ihre Umwelt von jener Haltung gegenüber dem Anderen bewusst aber zum größten Teil auch unbewusst geprägt ist. 


\section{Abschließende Bemerkung}

In der Einleitung wurde erwähnt, dass es ein Zeitalter geben wird, in dem es keine Holocaust-Zeitzeugen mehr geben wird, die dann auch nicht mehr ihre Geschichte erzählen und Aufarbeitungsarbeiten leisten können. Dieser Beitrag sollte ein Überblick leisten, wieviel bereits getan wurde und stets am Laufen ist. Die Holocaust-Zeitzeugen erzählen in den Videoaufzeichnungen der ARD- und ZDF-Mediathek aus eigener Erfahrung das Grauen des Nationalsozialismus' der Hitler-Diktatur. Diese Videoaufzeichnungen sind im Internet über Medien, aber vor allem den sozialen Medien abrufbar. Die doppelte Bedeutung und Funktion jener liegt - wie bereits dargelegt - darin, dass Jugendliche in den sozialen Medien sehr präsent sind. Der emotionale Bezug kann durch die Videoaufzeichnungen im Zeitalter des Kulturwandels erzeugt werden, da der erzählte Inhalt eine realhistorische Gegebenheit ist. Der Nachteil - wenn man es so sagen darf - dieser Videoaufzeichnungen besteht darin, dass, vergleichbar mit realen Zeitzeugen, der emotionale Bezug nicht so stark ist, denn wenn reale Personen am Erzählen der eigenen Geschichte sind, so ist die Berichterstattung aus erster Quelle und der Einfluss bei den Rezipienten umso stärker. Um aber den Grad der Intensität vom emotionalen Bezug auch in einem Zeitalter ohne Zeitzeugen hochzuhalten und/ oder zu erhöhen und die Rezipienten an die Gräueltaten zu erinnern, müssen technologische Innovationen mithilfe modernster Technik erfunden werden. Erste Projekte, die auch hier vorgeführt wurden, sind als Ansätze zu verstehen, wodurch der Versuch unternommen wird virtuelle Holocaust-Zeitzeugen zeitunabhängig zu reproduzieren. Demnach steigert man nicht nur die Intensitätsrate und hat einen größeren Erfolg in den Aufklärungsarbeiten, sondern man sorgt gleichzeitig dafür, dass einerseits mithilfe von Smartgeräten ein Zugang zu Videos und Audioaufzeichnungen gewährleistet wird und andererseits mithilfe modernster Technologie und Hologramm-Projektionen der Holocaust-Zeitzeugen den Kindern und Jugendlichen die Möglichkeit einer virtuellen Unterhaltung gegeben wird. Diese digitalen Zeugnisse sind authentisch und beispielsweise vergleichbar mit den Schulbesuchen, den die Holocaust-Zeitzeugen aktiv zu Lebzeiten immer wieder durchgeführt haben, aber es sind keine eins-zu-eins Abbildungen der nationalsozialistischen Geschichte. Das außergewöhnliche Leben einer Person, im vorliegenden Fall der Zeitzeugen, weckt umso mehr das Interesse bei Kindern und Jugendlichen, da nicht alle ein reges Interesse am Geschichtsunterricht oder den büchern haben.

Es hat sich zudem herausgestellt, dass das Anne Frank Lernlabor eher und primär mit diskriminierenden Faktoren arbeitet und in der Hinsicht Aufklärungsarbeit leistet. Kinder und Jugendliche, aber auch Erwachsene sollen durch aktives Lernen am eigenen Leibe erfahren, dass man Vorurteile gegenüber dem Anderen haben kann. Demzufolge wird der Fokus auf die inneren Werte gelenkt, die man gegebenenfalls modifizieren und revidieren muss. Die Lenkung auf diverse Themen der Diskriminierung soll dann eine Sensibilisierung ermöglichen, sodass eine möglichst vorurteilsfreie Zukunft erreicht werden kann. Neben den Aufklärungsarbeiten wäre es ebenfalls angebracht in den Medien sich intensiver den Leistungen der Juden vor der Shoah zu widmen. Dieser Aspekt fehlt zum größten Teil in der medialen Berichterstattung. Der Fokus liegt primär 
in der medialen Aufzeichnung des Lebens der Holocaust-Zeitzeugen und ihre Botschaft für zukünftige Generationen.

Abschließend kann gesagt werden, dass die ganzen Bemühungen und Bestrebungen das Ziel vor Auge haben, ein psychologisches Mahnmal zu setzen, sodass die Gräueltaten nicht in Vergessenheit geraten und Auschwitz sich nicht wiederholt. Der Akt des NichtVergessens und somit des Erinnerns gilt als das höchste Gebot. Von der Schuldfrage sind Folgegenerationen freigesprochen, so dass die Grundlage für eine friedliche Zukunft ermöglicht wird. Die in diesem Jahr (2021) verstorbene Holocaust-Zeitzeugin Esther Bejarano, die im Mädchenorchester der nationalsozialistischen Diktatur in Auschwitz wider Willen Akkordeon spielen musste und in der Nachkriegszeit sehr aktiv an den Aufklärungsarbeiten mitgewirkt hat und ihre Hoffnung in die Jugend setzte, bringt den Schuldfreispruch an den Tag: ,Ihr seid nicht schuld an dieser schrecklichen Zeit, aber ihr macht euch schuldig, wenn ihr nichts über die Geschichte wissen wollt" (Große-Wilde 2021).

\section{Literaturverzeichnis}

Assmann, Jan (2013): Das kulturelle Gedächtnis: Schrift, Erinnerung und politische Identität in frühen Hochkulturen. München: Beck.

Ballis, Anja (2020): Die Migrationsgesellschaft als Herausforderung für Holocaust Education im Jugendund Erwachsenenalter. In: Bildung und Erziehung. 3, 226-241.

Ballis, Anja (2021): Interaktive 3D-Zeugnisse von Holocaust-Überlebenden im Deutschunterricht Theoretische Rahmung, empirische Exploration und disziplinäre Zielhorizonte. In: Ballis, Anja et al. (Hrsg.): Interaktive 3D-Zeugnisse von Holocaust-Überlebenden. Chancen und Grenzen einer innovativen Technologie. Eckert. Dossiers. 1, 83-110.

Ballis, Anja / Gloe, Markus (2020): Interactive 3D testimonies of Holocaust survivors in German language. Methodological framework for resarch and education. In: Gloe, Markus und Anja Ballis (Hrsg.): Holocaust Education Revisited. Wiesbaden: Springer VS, 343-364.

Ballis, Anja / Barricelli, Michele / Gloe, Markus (2019): Interaktive digitale 3-D-Zeugnisse und Holocaust-Education - Entwicklung, Präsentation und Erforschung. In: Ballis, Anja und Markus Gloe (Hrsg.): Holocaust Education Revisited. Wahrnehmung und Vermittlung. Fiktion und Fakten. Medialität und Digitalität. Wiesbaden: Springer VS, 403-436.

Boudon, Raymond (1979): La logique du social. Introduction à l'analyse sociologique. Paris: Hachette Littérature.

Coşan, Leyla / Tekin, Habib (2021): Stefan Zweigs Werke im Lichte der jüdischen Erinnerungskultur. In: Coşan, Leyla und Mehmet Tahir Öncü (Hrsg.): Jüdische Lebenswelten im Diskurs. Berlin: Logos, 125-145.

Coşan, Leyla / Tekin, Habib (2021): Bilder der Judenfeindschaft in den Werken von Jakob Wassermann. In: Diyalog. Interkulturelle Zeitschrift für Germanistik. 2021/ 1, 3-22.

Duda, Florian (2021): Was war oder ist Ihre schönste, tollste und angenehmste Kindheitserinnerung? Ein sprachwissenschaftlicher Ansatz zur Machine-Learning-Datengenerierung. In: Ballis, Anja et al. (Hrsg.): Interaktive 3D-Zeugnisse von Holocaust-Überlebenden. Chancen und Grenzen einer innovativen Technologie. Eckert. Dossiers 1, 43-62. 
Eckmann, Monique (2006): Rassismus und Antisemitismus als pädagogische Handlungsfelder. In: Fechler, Bernd, Gottfried Kößler, Astrid Messerschmidt, Barbara Schäuble (Hrsg.): Neue Judenfeindschaft - Perspektiven für den pädagogischen Umgang mit dem globalisierten Antisemitismus. Frankfurt am Main: Campus, 210-232.

Elleström, Lars (2018): A medium-centered model of communication. In: Semiotica. 224, 269-293.

Fromm, Erich (1977): Anatomie der menschlichen Destruktivität. Reinbek: Rowohlt.

Fuchs-Heinritz, Werner et al. (Hrsg.) (1981): Lexikon zur Soziologie. Berlin: Springer VS.

Gloe, Markus (2021): Digital Interactive 2D/ 3D Testimonies in Holocaust Museums in the United States and Europe. In: Ballis, Anja et al. (Hrsg.): Interaktive 3D-Zeugnisse von Holocaust-Überlebenden. Chancen und Grenzen einer innovativen Technologie. Eckert. Dossiers 1, 130-146.

Groh, Arnold (2004): Kulturwandel durch Reisen: Faktoren, Interdependenzen, Dominanzeffekte. In: Christian Berkemeier, Katrin Callsen, Ingmar Probst (Hrsg.): Begegnung und Verhandlung: Möglichkeiten eines Kulturwandels durch Reise. LIT Verlag: Münster, 13-31.

Heindl, Fabian (2021): The Role of Narrative Structures and Contextual Information in Digital Interactive 3D Testimonies. In: Ballis, Anja et al. (Hrsg.): Interaktive 3D-Zeugnisse von HolocaustÜberlebenden. Chancen und Grenzen einer innovativen Technologie. Eckert. Dossiers 1, 111-129.

Immerfall, Stefan (1991): Sozialer Wandel in der Moderne. Neuere Forschungsergebnisse zum Prozeß gesellschaftlicher Modernisierung im 19. und 20. Jahrhundert. In: Neue politische Literatur, 36, 548 .

Kolb, Daniel (2021): Evaluation of the Interaction with a Digital 3D Testimony - Between Emotion and Technology. In: Ballis, Anja et al. (Hrsg.): Interaktive 3D-Zeugnisse von Holocaust-Überlebenden. Chancen und Grenzen einer innovativen Technologie. Eckert. Dossiers 1, 63-82.

Krämer, Sybille (2008): Medium, Bote, Übertragung. Kleine Metaphysik der Medialität, Frankfurt am Main: Suhrkamp.

Lévi-Strauss, Claude (1975): Strukturale Anthropologie II. Frankfurt am Main: Suhrkamp.

Reichertz, Jo (2009): Kommunikationsmacht. Was ist Kommunikation und was vermag sie? Und weshalb vermag sie das?, Wiesbaden: Verlag für Sozialwissenschaften.

Schwendemann, Lisa / Hütl, Ernst (2021): 1000 Fragen an Abba Naor und Eva Umlauf - Die Rolle der Fragen und Antworten für die Erstellung interaktiver 3D-Zeugnisse. In: Ballis, Anja et al. (Hrsg.): Interaktive 3D-Zeugnisse von Holocaust-Überlebenden. Chancen und Grenzen einer innovativen Technologie. Eckert. Dossiers 1, 29.

Demirkıvıran, Sine (2021): Mirjam Pressler „Nathan und seine Kinder“: Adaptation und Rewriting für junge Leser. In: Coşan, Leyla / Öncü, Mehmet Tahir (Hrsg.): Jüdische Lebenswelten im Diskurs. Berlin: Logos, 223-247.

Tekin, Habib (2021): Bilder der Judenfeindschaft in den Werken von Jakob Wassermann. Berlin: Logos.

\section{Internetquellen}

Bildungsstätte Anne Frank (k.A.): Mensch, du hast Recht(e). Das mobile Lernlabor. Eine Wanderausstellung der Bildungsstätte Anne Frank. (Reader). https://www.bs-annefrank.de/fileadmin/content/Publikationen/Weiteres_P\%C3\%A4dagogisches_Material/Mensch_du_ hast_Rechte_Reader_A4.pdf. (Letzter Zugriff: 31.10.2021).

Gloe, Markus (2020): Interaktive digitale Zeugnisse von Holocaust-Überlebenden: Eine innovative Technologie im Einsatz an Schulen und Hochschulen. https://www.qualitaetsoffensive- 
lehrerbildung.de/lehrerbildung/de/newsletter/_documents/interaktive-digitale-zeugnisse-atz-anschulen-und-hochschulen (Letzter Zugriff: 31.10.2021).

Große-Wilde, Carola (2021): KZ-Überlebende Esther Bejarano ist tot. Wichtige Stimme gegen das Vergessen. https://www.zdf.de/nachrichten/politik/esther-bejarano-holocaust-ueberlebende-tot100.html (Letzter Zugriff: 31.10.2021).

KMK (2016): Strategie der Kultusministerkonferenz „Bildung in der digitalen Welt“. https://www.kmk.org/fileadmin/Dateien/pdf/PresseUndAktuelles/2017/Strategie_neu 2017_datum_1.pdf. (Letzter Zugriff: 31.10.2021).

\section{Abbildungsverzeichnis}

Abb. 1: ARD Zukunftspodcast über die Holocaust-Zeitzeugen

Abb. 2: Kommunikationsmodell

Abb. 3: An 3D-Zeugnisse angepasstes Kommunikationsmodell in Anlehnung an Elleström

Abb. 4: Jugendlicher (rechts) vor einem Bodyscanner

Abb. 5: Station Schublade mit Titelbildern

Abb. 6: Wahlplakat der Grünen

Abb. 7: Coverbild der Zeitschrift Der Spiegel

Abb. 8: Etikett der Heinz Ketchup 